OPEN ACCESS

Edited by:

Wenbo Luo,

Liaoning Normal University, China

Reviewed by:

Gianluca Serafini,

San Martino Hospital, University of

Genoa, Italy

Yiqun Wang,

Fudan University, China

*Correspondence:

Bingjin $\mathrm{Li}$

libingjin@jlu.edu.cn

Xuewen Zhang

zhangxw@jlu.edu.cn

Received: 19 August 2017 Accepted: 18 October 2017

Published: 06 November 2017

Citation:

Liu S, Sheng J, Li B and Zhang X (2017) Recent Advances in Non-invasive Brain Stimulation for Major Depressive Disorder.

Front. Hum. Neurosci. 11:526. doi: 10.3389/fnhum.2017.00526

\section{Recent Advances in Non-invasive Brain Stimulation for Major Depressive Disorder}

\author{
Shui Liu, Jiyao Sheng, Bingjin Li * and Xuewen Zhang * \\ Jilin Provincial Key Laboratory on Molecular and Chemical Genetics, The Second Hospital of Jilin University, Changchun, \\ China
}

Non-invasive brain stimulation (NBS) is a promising treatment for major depressive disorder (MDD), which is an affective processing disorder involving abnormal emotional processing. Many studies have shown that repetitive transcranial magnetic stimulation (rTMS) and transcranial direct current stimulation (tDCS) over the prefrontal cortex can play a regulatory role in affective processing. Although the clinical efficacy of NBS in MDD has been demonstrated clinically, the precise mechanism of action remains unclear. Therefore, this review article summarizes the current status of NBS methods, including rTMS and tDCS, in the treatment of MDD. The article explores possible correlations between depressive symptoms and affective processing, highlighting the relevant affective processing mechanisms. Our review provides a reference for the safety and efficacy of NBS methods in the clinical treatment of MDD.

\footnotetext{
Keywords: non-invasive brain stimulation, repetitive transcranial magnetic stimulation, transcranial direct current stimulation, affective processing, major depressive disorder
}

\section{INTRODUCTION}

Major depressive disorder (MDD) is one of the most common and disabling mental disorders, and has become the second leading contributor to the global disease burden (Collins et al., 2011; Whiteford et al., 2013; Otte et al., 2016). MDD is characterized by maladaptive and persistent emotional responses to stressors (Groenewold et al., 2013). Because of its high incidence and common recurrence, MDD represents a serious challenge for world public health. Currently, approximately $1 / 3$ of MDD patients globally exhibit treatment-resistant depression, because of invalid or ineffective antidepressant treatment (Rush et al., 2006).

Affective processing is crucial for the basic tasks of human survival and adaptation, involving many functions including perception, attention, learning, memory and responses to the environment (Narumoto et al., 2001; Garrett and Maddock, 2006; Del Piero et al., 2016). Dysfunctional affective processing is considered a key factor in the occurrence and development of many psychiatric disorders, including anxiety, schizophrenia and bipolar disorder (Anderson et al., 2017; Bocharov et al., 2017; Krakowski and Czobor, 2017; Wolkenstein et al., 2017). Previous studies have suggested that MDD is associated with dysfunctional processing in affective-related neural circuits (Clark et al., 2009). Meanwhile, cognitive abnormalities are also a core feature of depression, which involves many domains including attention, memory, executive functions and psychomotor speed (Gonda et al., 2015). Beck proposed a cognitive model of depression 


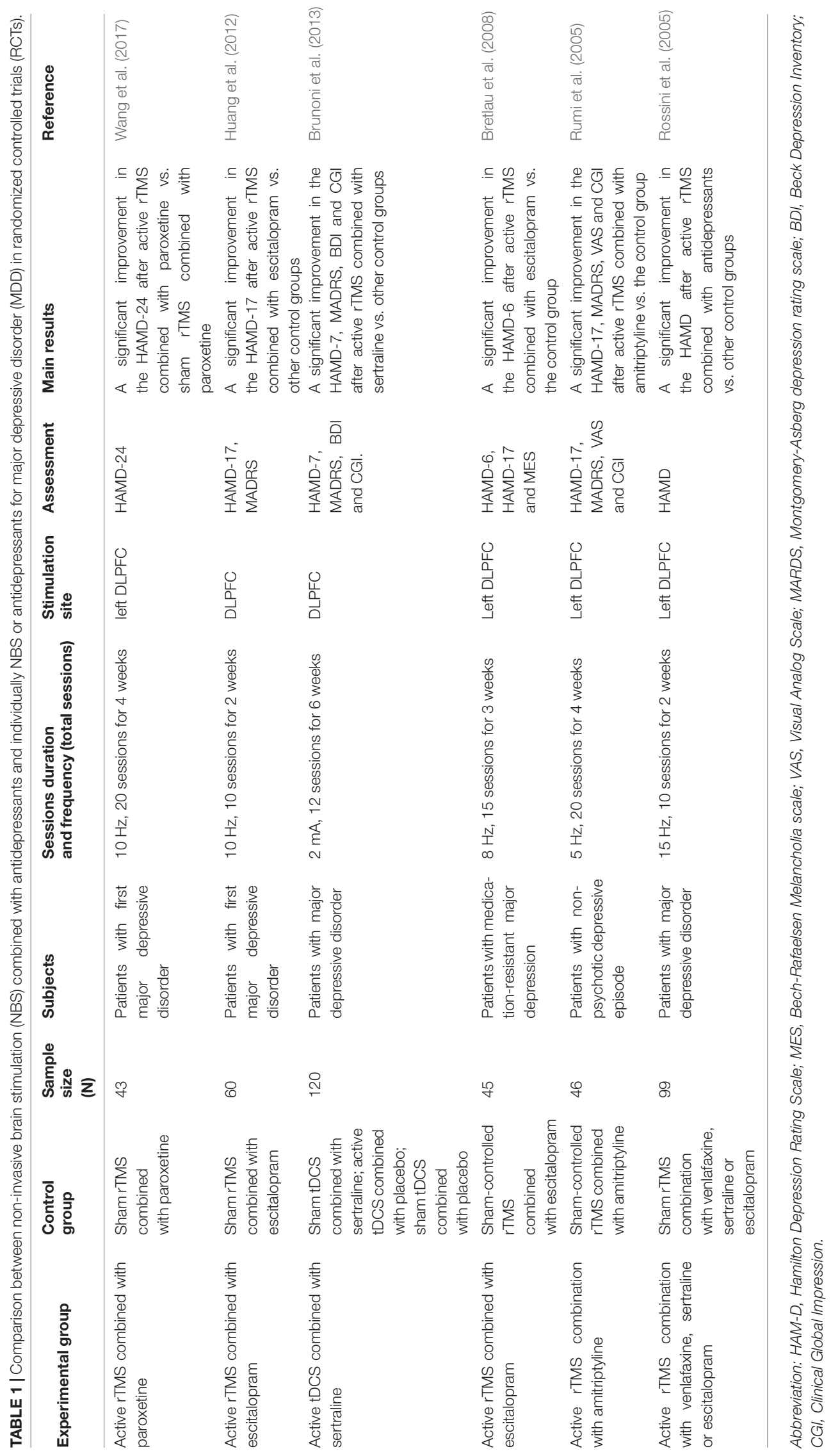




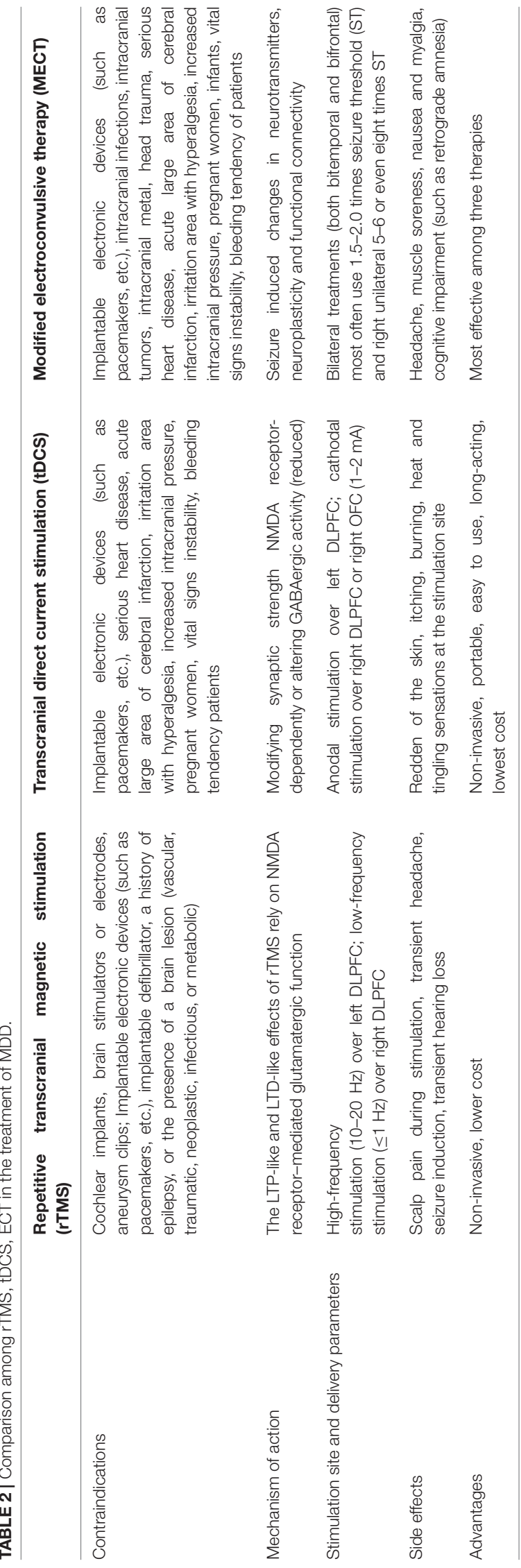

in which negative stimuli in the environment can attract conscious or unconscious attention, and patients with depression tend to make negative evaluations of themselves and others, suggesting that depression might be caused by negative cognitive schemas (Disner et al., 2011). For example, hopelessness, manifested as overestimating the damage of a negative event and underestimating the positive outcome of the future, is thought to be an important cognitive risk factors of depression (Wang et al., 2015). Many behavioral studies have demonstrated that patients with MDD exhibit a negative emotional bias, manifesting as preferential processing of negative over positive stimuli, in accord with Beck's hypothesis (Erickson et al., 2005; Leyman et al., 2007; Yang et al., 2011). Strunk and Adler (2009) examined the relationship between depressive symptoms and bias, reporting that patients high in depressive symptoms exhibited significant pessimistic bias on three judgment tasks. The modern cognitive neuropsychological model of depression is a reformulation and expansion of Beck's cognitive model of depression, and the results are derived from pharmacological studies and concerning basic neurocognitive function. This model also proposes that patients with depression may develop an alteration in the bottom-up emotional stimulus processing, leading to negative perception (Roiser et al., 2012; Gonda et al., 2015).

Recent evidence suggests that negative affective and cognitive processing bias of MDD patients may originate from structural and functional abnormalities in specific brain regions, including the dorsolateral and ventral prefrontal cortex, hippocampus and amygdala, which are associated with affective processing (Campbell et al., 2004; Hamilton et al., 2008; Koenigs and Grafman, 2009). Numerous studies have demonstrated that the networks abnormality is one crucial mechanism in the occurrence and development of MDD, which underlies altered affective and cognitive processing, such as increased reactivity as well as increased attentional and cognitive bias towards negative stimuli in MDD (Hamilton et al., 2012; Groenewold et al., 2013). And the default mode network (DMN), the executive control network (ECN), and the salience network (SN) are three major networks in the recent studies of MDD. The DMN is involves in self-referential processing and episodic memory retrieval (Raichle et al., 2001). The ECN is involved in executive function and emotion regulation, with functional regions being the dorsolateral prefrontal cortex (DLPFC) and lateral posterior parietal regions (Miller and Cohen, 2001). The core regions in the SN include amygdala, and anterior hippocampus, which is involved in detection of, and direction of attention to, salient environmental stimuli (Chen and Etkin, 2013). Some studies revealed functional connectivity changes in the ECN and SN in depressed patients (Bonavita et al., 2017), which suggested the potential links between networks abnormality and depressive symptoms. Studies found that the enhancement of affective processing in MDD manifested excessive activation of the amygdala and supragenual cingulate of the brain (Matthews et al., 2008; Davey et al., 2011). In addition, the deficiency of cognitive control in MDD has demonstrated 
the correlations with insufficient activity in the DLPFC and anterior cingulate gyrus (Holmes and Pizzagalli, 2008; McNeely et al., 2008). These findings suggest that during the affective processing of depressive individuals, networks abnormalities, manifested as enhanced affective processing and decreased cognitive control function, might result in a more intense experience of negative emotion, inducing depression.

Non-invasive brain stimulation (NBS) methods have been found to be effective for regulating human brain function, including transcranial magnetic stimulation (TMS), transcranial direct current stimulation (tDCS) and transcranial alternating current stimulation (tACS). In the past few decades, NBS techniques have been found to be useful for regulating healthy individual control of consciousness, and positive therapeutic effects have been reported for a range of psychiatric disorders, including depression, Alzheimer's disease, and epilepsy (Kuo et al., 2014; De Raedt et al., 2015). As NBS techniques develop, they have been applied in the clinical treatment of MDD, and the regulatory effects of NBS on affective processing have been consistently verified (Nitsche et al., 2012; Conson et al., 2015).

In the current review, we discuss the possible mechanisms by which NBS methods, including repetitive TMS (rTMS) and tDCS, improve depressive symptoms by modulating affective processing. Moreover, we examine research investigating the value of combining NBS with imaging techniques to improve antidepressant effects. Thus, this review article can provide a reference for the safety and efficacy of NBS methods in the clinical treatment of MDD.

\section{REPETITIVE TRANSCRANIAL MAGNETIC STIMULATION (rTMS)}

\section{rTMS Overview}

TMS is an NBS technique that was first created in 1985 by Brunoni et al. (Valero-Cabré et al., 2017). In TMS, electromagnetic induction is used to focus a current and modulate cortical function (Hallett, 2007). TMS devices consist of a capacitor to store charge and a stimulation coil to transfer energy. When the charge capacitor is rapidly released, the generated current passes through the stimulating coil to produce magnetic lines of flux with low resistance and no trauma, penetrating the skull to reach the cortex and reverse the current conduction in the cortex, thereby altering cortical excitability (Noda et al., 2015). rTMS is a new neurophysiological technique based on TMS, involving the delivery of repetitive stimuli at a specific cortical site. Previous studies have shown that low-frequency stimulation of rTMS $(\leq 1 \mathrm{~Hz})$ can reduce the excitability of neurons and inhibit cortical activity, whereas high-frequency stimulation $(\geq 5 \mathrm{~Hz}$ ) can increase the excitability of neurons and enhance cortical activity (Mitchell and Loo, 2006; Milev et al., 2016). To date, rTMS has been approved as a clinical therapy for MDD in several regions, including the USA, Canada the European Union (Tortella et al., 2014).

\section{Affective Processing-Related Mechanisms of rTMS in Antidepressant Treatment}

Previous studies have indicated that multiple brain regions, including the amygdala, prefrontal cortex, parietal lobe and modality-specific sensory cortex regions, play distinct roles in regulating emotional processing (Dalgleish, 2004; Pessoa, 2017). The valence hypothesis proposes that affective processing exhibits hemispheric lateralization, with the right hemisphere specializing in negative emotion processing and the left hemisphere specializing in positive emotion processing (Prete et al., 2015). This hypothesis has been supported by neuroimaging studies (Grimm et al., 2008), and several previous studies have shown that the DLPFC influences emotional stimulus categorization, emotional evaluation, emotional memory, and emotional regulation (Brennan et al., 2017; Zilverstand et al., 2017). Thus, the DLPFC is thought to play a leading role in emotional control. Previous studies reported that activation of the left DLPFC is associated with processing positive emotions, whereas activation of the right DLPFC is thought to be responsible for processing negative emotions (Mondino et al., 2015). Schutter and van Honk (2005) found that, in patients with depression, left DLPFC responses were decreased and right DLPFC responses were increased.

rTMS is a relatively localized intervention, and several studies have examined its potential role in antidepressant treatment, with the DLPFC as a primary target (Lepping et al., 2014; Serafini et al., 2015; Carle et al., 2017; Carpenter et al., 2017). Two rTMS protocols are commonly used for treating MDD: high-frequency rTMS $(10-20 \mathrm{~Hz})$ targeting left DLPFC, and low-frequency rTMS $(\leq 1 \mathrm{~Hz})$ targeting right DLPFC (Isenberg et al., 2005). Zwanzger et al. (2014) reported that inhibitory rTMS over the right DLPFC could improve and regulate affective processing, indicating that rTMS might exert an antidepressant role via affective processing-related mechanisms. A study of the role of frontal stimulation in emotional processing by Vanderhasselt et al. (2009) revealed that high-frequency rTMS applied to left DLPFC can improve task-switching abilities in depressed individuals. Moreover, clinical evidence has indicated that low-frequency rTMS over right DLPFC can increase response rates to monotherapy for MDD (Berlim et al., 2013b). These findings are consistent with the valence hypothesis, providing strong support for the notion that the antidepressant effects of rTMS involve the regulation of affective processing.

At the same time, some other brain regions associated with affective processing, including dorsomedial prefrontal cortex (DMPFC), frontopolar cortex (FPC), ventromedial prefrontal cortex (VMPFC), and ventrolateral prefrontal cortex (VLPFC), have been also considered as potential targets for clinical application of rTMS (Downar and Daskalakis, 2013; Junghofer et al., 2017). Of these, DMPFC received the most attention to date. Bakker et al. (2015) found that DMPFC-rTMS could show a similar antidepressant effect of DLPFC-rTMS in patients with MDD. Case series in MDD and bipolar disorder have provided initial evidence that DMPFC-rTMS may be safe, tolerable and effective in antidepressant treatment (Downar and Daskalakis, 2013; Downar et al., 2014). Nevertheless, 
there is a need for more researches and clinical trials of DMPFC as a target for clinical application of rTMS in MDD.

\section{Combination of rTMS and Antidepressants in the Treatment of MDD}

Previous studies have indicated that rTMS can lead to long-term and sustained remission of treatment-resistant MDD, significantly improving the quality of life and functional status of MDD patients (Galletly et al., 2016; Teng et al., 2017). Moreover, some studies have found that rTMS may improve antidepressant effect in combination with traditional antidepressants (Table 1). In a study by Wang et al. (2017), 43 patients with first-episode MDD were randomly divided into two groups. Subsequently, active or sham rTMS was applied to the left DLPFC, and a 4-week course of combination therapy with paroxetine was administered. The results indicated that patients in the active rTMS group had a higher response rate than those in the sham rTMS group at the end of the fourth week, and the remission rate in the experiment group was clearly elevated compared with the control group (Wang et al., 2017). These results suggest that rTMS might enhance the response of depressed patients to paroxetine, enhancing antidepressant efficacy. A double-blind clinical randomized controlled trial (RCT) by Huang et al. (2012) also confirmed the efficacy of rTMS in combination with conventional antidepressants in the treatment of depression. In their study, 60 patients with first-episode MDD were randomly categorized into two groups. In the first 2 weeks, patients in the two groups were treated with active or sham rTMS combined with escitalopram treatment, followed by another 2 weeks of escitalopram monotherapy. The results revealed that, compared with the control group, scores on the 17-item Hamilton Depression Rating Scale (HAMD-17) dropped more than $20 \%$ in the active rTMS group in the first 2 weeks. Furthermore, the active rTMS group exhibited a significantly faster score reduction compared with the sham group at 2 weeks, suggesting that rTMS had a synergistic effect in the treatment of MDD with traditional antidepressants.

\section{Comparison of rTMS and ECT in Antidepressant Treatment}

Electroconvulsive therapy (ECT) has been used for the treatment of human diseases for more than 80 years, and is currently considered the most effective treatment for MDD (UK ECT Review Group, 2003). At present, the main technique used in clinical settings is modified ECT (MECT). This method involves the administration of anesthetics and muscle relaxants before treatment, so that the electrical stimulation does not cause convulsions, which in turn results in the elimination of muscle rigidity and tremor, as well as avoiding fracture, dislocation and other complications (Liu et al., 2016). Although ECT has been shown to be effective in the short term, its recurrence rate, particularly the high rate of early recurrence, and the cognitive side effects are important challenges in this form of antidepressant treatment (Jelovac et al., 2013; Fernie et al., 2014). A meta-analysis reported that despite continued drug treatment after ECT treatment, the relapse rates was $51.1 \%$ in the first year after treatment, peaking in the first 6 months, up to $37.7 \%$ (Jelovac et al., 2013).

There are clear differences in antidepressant mechanism, tolerance and acceptability between rTMS and ECT (Table 2). The antidepressant effects of rTMS and ECT in MDD have been compared in numerous studies (Möbius et al., 2017). Chen et al. (2017) conducted a meta-analysis including 25 clinical RCTs involving 1288 MDD patients. The findings revealed that the therapeutic effects of ECT were greater than those of rTMS, but right prefrontal-rTMS had the best tolerance. Jin et al. (2016) performed a retrospective study of $150 \mathrm{MDD}$ patients receiving MECT and 150 MDD patients receiving rTMS, showing that in the short-term, the response rate in the MECT group was higher than that in the rTMS group, although there was no clear difference in long-term relapse-free survival between groups. Furthermore, the cost benefit of ECT was found to be higher than that of rTMS, and, because of its non-invasive and convenient features, as well as its minimal side effects relative to ECT, rTMS was favored by patients (Magnezi et al., 2016). Nevertheless, as an emerging treatment technology for antidepressant therapy, further in-depth clinical studies of rTMS are required before it becomes a widespread alternative to ECT.

\section{Disadvantages and Side Effects}

The most common side effects of rTMS in clinical settings include headache $(5 \%-23 \%)$ and discomfort at the stimulus site $(20 \%-40 \%)$, and the most severe side effect is the induction of seizures (Machii et al., 2006; Maizey et al., 2013; Wall et al., 2014; Dobek et al., 2015; Boes et al., 2016). Prikryl and Kucerova (2005) reported a case of generalized tonic clonic seizure in a patient with MDD receiving rTMS. To date, fewer than 25 cases of rTMS-induced seizure have been reported worldwide. Therefore, high frequency rTMS is contraindicated in patients with a history of seizures, although the incidence rate is relatively low $(<0.1 \%$; Dobek et al., 2015).

\section{TRANSCRANIAL DIRECT CURRENT STIMULATION (tDCS)}

\section{tDCS Overview}

tDCS is an NBS method acting on specific cortical areas by producing a persistent, weak, direct current (usually 1-2 mA) through electrodes placed on the skull (Blumberger et al., 2015). The basic principle is that stimuli with different polarities can cause changes in the hyperpolarization or depolarization of the resting membrane potential. Anodic stimulation can improve the excitability of the cortex through the depolarization of the membrane potential, while cathodic stimulation can help reduce cortical excitability via hyperpolarization of neuronal membrane potentials (Stagg and Nitsche, 2011). Previous studies have indicated that the neurophysiological mechanisms of tDCS may involve subliminal regulation of the resting membrane potential of neurons inducing a polarity-dependent modification of 
$\mathrm{N}$-methyl-d-aspartate (NMDA) receptor function (Nitsche et al., 2003). Because NMDA receptor function is involved in synaptic plasticity formation, this can result in the production of neural remodeling and changes in the excitability of the cortex during stimulation (Nitsche et al., 2003). The stimulation of tDCS is weak, but it can also cause changes in cortical excitability, and the effect lasts longer after stimulation than that of rTMS. A previous study reported that following current stimulation of the body for several minutes, changes in cortical excitability can last for approximately $1 \mathrm{~h}$ after stimulation (Nitsche and Paulus, 2000). Meanwhile, compared with rTMS, tDCS has the advantages of portability, low equipment cost and minimal adverse reactions (Lefaucheur et al., 2017; Table 2).

\section{Affective Processing-Related Mechanisms of tDCS in Antidepressant Treatment}

The DLPFC is one of the major brain areas involved in emotion regulation (Baeken et al., 2010), and various neuroimaging studies have indicated that it plays an important role in top-down regulation of affective processing (Disner et al., 2011). Some studies have suggested that DLPFC activity can be mediated by tDCS, thus playing a regulatory role in affective processing (Boggio et al., 2009; Sanchez et al., 2016). Single-session tDCS studies in healthy samples by Utz et al. (2010) revealed acute improvement in affective and cognitive processing. Further research has confirmed that the DLPFC plays an important role in the occurrence and development of depression (Schutter and van Honk, 2005). Imaging studies have also shown that left DLPFC cerebral blood flow and metabolism are decreased in patients with depression, while the right DLPFC exhibits increased metabolic activity (Shiozawa et al., 2015).

A number of studies have confirmed the role of tDCS in antidepressant treatment (Vigod et al., 2014; Al-Kaysi et al., 2017; Brennan et al., 2017). At present, the left and right DLPFC are typically used as anode and cathode stimulation sites for the majority of tDCS treatment methods, which can increase the excitability of the left DLPFC and inhibit the excitability of the right DLPFC to alleviate depressive symptoms (Meron et al., 2015). Through a double-blind RCT, Wolkenstein and Plewnia (2013) detected the effect of a single-session anodal tDCS targeting the left DLPFC in MDD patients, reporting a significant improvement in emotional cognitive control. This finding provided further evidence that tDCS might improve depressive symptoms by modulating emotional processing. Brunoni et al. (2011, 2013, 2014) conducted a double-blind RCT involving 24 depressive patients, and presented the emotional Stroop task, measuring response times (RTs) to positive-, negative-, and neutralrelated words. The results revealed that active tDCS significantly modified negative attentional bias, abolishing the RT delay for negative words (Brunoni et al., 2014). This finding suggests that the regulatory effect of tDCS on affective processing might be an important mechanism underlying the antidepressant effects of the treatment method.

\section{Combination of tDCS and Antidepressants in the Treatment of MDD}

Some studies have found that $\mathrm{tDCS}$ combined with traditional antidepressants might have a synergistic therapeutic effect (Table 1). Brunoni et al. (2011, 2013, 2014) conducted a double-blind RCT, dividing participants into four groups using pairwise combinations of sertraline/placebo and active/sham tDCS. When Montgomery-Asberg Depression Rating Scale (MADRS) scores were measured, the results revealed that combined treatment was significantly superior to placebo, tDCS only, and sertraline only. There was no significant difference in side effects between different modalities of intervention (Brunoni et al., 2013), indicating that the combination of tDCS and antidepressants in patients with MDD performed better than applying either treatment alone. These findings may provide a new direction for the widespread application of tDCS in MDD treatment.

\section{Disadvantages and Side Effects}

According to the current safety guidelines of tDCS, the adverse effects are minimal for both healthy individuals and MDD patients, regardless of whether tDCS is applied to the motor areas or non-motor areas of the cortex. Reddening of the skin, heat, burning, itching, and tingling sensations at the stimulation site are the most common side effects of the treatment, and are reported by more than half of patients receiving tDCS (Brunoni et al., 2011; Shiozawa et al., 2014; Meron et al., 2015). Brunoni et al.'s (2011) systematic review of 117 studies conducted between 1998 and 2010 investigated the adverse effects of tDCS on the human brain, reporting that slight itching and tingling were the main adverse events, and that retention time was transient.

\section{PROSPECTS FOR NBS TECHNIQUES IN ANTIDEPRESSANT TREATMENT}

The efficacy of NBS in treatment remains limited, even though its effectiveness in improving depressive symptoms in MDD patients has been consistently validated (McLoughlin et al., 2007; Berlim et al., 2013a; Lefaucheur et al., 2017). As a local brain stimulation technique, the therapeutic efficacy of NBS depends largely on the choice of stimulation sites and the accuracy of the location (Herbsman et al., 2009; Fox et al., 2012). Several previous studies have indicated that imaging-guided NBS could help to locate specific functional brain networks at a higher resolution. Using this approach, stimulation sites can be individually and accurately positioned according to anatomical differences of individual depressed patients, thereby improving the therapeutic effectiveness of NBS in antidepressant treatment, and supporting the extensive application of NBS approaches in the clinic (Mir-Moghtadaei et al., 2015; Luber et al., 2017).

Jha et al. (2016) examined the effects of a 4-week treatment regime in refractory MDD patients with singlephoton emission computed tomography (SPECT) guided high-frequency rTMS and standard high-frequency rTMS. In 
their experiment, subjects were assessed with the MADRS, the Beck Depression Inventory (BDI) and the Clinical Global Impression (CGI) scale. The response rate of the subjects in the brain SPECT guided group was found to be significantly higher than that in the standard group, on MADRS, BDI and CGI scores (Jha et al., 2016). These findings indicate that rTMS combined with brain SPECT targeting specific brain regions could improve antidepressant treatment in clinical settings.

An important topic for the future development of NBS is determining the combinations of imaging methods that provide optimal antidepressant treatment effects, to develop individualized treatment for MDD patients.

\section{LIMITATIONS}

Several limits of this systematic review should be acknowledged. First, a common limitation to the research presented in this review is the widespread differences in measurement tools used to measure depressive symptoms and identify depression. A large number of different scales for measuring depressive symptoms were used across different studies, including the HAM-D, MES, VAS, MARDS, BDI and CGI, which may produce different amounts of measurement error in different samples depending on the population in which they are being used. Second, sample size of some RCTs in the review is relatively small. Finally, this review may be limited by reporting bias, the under-reporting of undesirable or non-significant experimental results. This may have leaded to lacking negative reports on the association between NBS and MDD, further weakening the evidence against a role of $\mathrm{NBS}$ in MDD.

\section{REFERENCES}

Al-Kaysi, A. M., Al-Ani, A., Loo, C. K., Powell, T. Y., Martin, D. M., Breakspear, M., et al. (2017). Predicting tDCS treatment outcomes of patients with major depressive disorder using automated EEG classification. J. Affect. Disord. 208, 597-603. doi: 10.1016/j.jad.2016. 10.021

Anderson, N. E., Steele, V. R., Maurer, J. M., Rao, V., Koenigs, M. R., Decety, J., et al. (2017). Differentiating emotional processing and attention in psychopathy with functional neuroimaging. Cogn. Affect. Behav. Neurosci. 17, 491-515. doi: 10.3758/s13415-016-0493-5

Baeken, C., De Raedt, R., Van Schuerbeek, P., Vanderhasselt, M. A., De Mey, J., Bossuyt, A., et al. (2010). Right prefrontal HF-rTMS attenuates right amygdala processing of negatively valenced emotional stimuli in healthy females. Behav. Brain Res. 214, 450-455. doi: 10.1016/j.bbr.2010. 06.029

Bakker, N., Shahab, S., Giacobbe, P., Blumberger, D. M., Daskalakis, Z. J., Kennedy, S. H., et al. (2015). rTMS of the dorsomedial prefrontal cortex for major depression: safety, tolerability, effectiveness, and outcome predictors for $10 \mathrm{~Hz}$ versus intermittent theta-burst stimulation. Brain Stimul. 8, 208-215. doi: 10.1016/j.brs.2014. 11.002

Berlim, M. T., Van den Eynde, F., and Daskalakis, Z. J. (2013a). Efficacy and acceptability of high frequency repetitive transcranial magnetic stimulation (rTMS) versus electroconvulsive therapy (ECT) for major depression: a systematic review and meta-analysis of randomized trials. Depress. Anxiety 30, 614-623. doi: 10.1002/da. 22060

\section{CONCLUSION}

As an emerging non-invasive antidepressant treatment approach with few adverse reactions, NBS techniques have been extensively studied since their inception, and their clinical application in the treatment of MDD is increasing. Studies have indicated that the development of MDD may be closely related to abnormal affective processing (Harmer et al., 2009). Brunoni et al. (2014) found that one single active bi-frontal tDCS significantly modifies negative attentional bias in MDD. Other studies found that NBS including tDCS and rTMS can improved deficient cognitive control, further enhancing affective processing in MDD (Hoy et al., 2012; Wolkenstein and Plewnia, 2013). In a word, NBS may alleviate the symptoms of depression by regulating affective processing and enhancing cognitive control. As research progresses, it is likely that the antidepressant mechanisms of NBS will become more specific, the corresponding treatment effects will continue to improve, and its applications in MDD treatment will become more extensive.

\section{AUTHOR CONTRIBUTIONS}

SL and JS wrote the manuscript. XZ and BL provided the critical revisions. All authors approved the final version of the manuscript for submission.

\section{ACKNOWLEDGMENTS}

The work was supported by the Graduate Innovation Fund of Jilin University and Norman Bethune Program of Jilin University (2015212).

Berlim, M. T., Van den Eynde, F., and Jeff Daskalakis, Z. (2013b). Clinically meaningful efficacy and acceptability of low-frequency repetitive transcranial magnetic stimulation (rTMS) for treating primary major depression: a meta-analysis of randomized, double-blind and sham-controlled trials. Neuropsychopharmacology 38, 543-551. doi: 10.1038/npp.20 12.237

Blumberger, D. M., Hsu, J. H., and Daskalakis, Z. J. (2015). A review of brain stimulation treatments for late-life depression. Curr. Treat. Options Psychiatry 2, 413-421. doi: 10.1007/s40501-015 -0059-0

Bocharov, A. V., Knyazev, G. G., and Savostyanov, A. N. (2017). Depression and implicit emotion processing: an EEG study. Neurophysiol. Clin. 47, 225-230. doi: 10.1016/j.neucli.2017.01.009

Boes, A. D., Stern, A. P., Bernstein, M., Hooker, J. E., Connor, A., Press, D. Z., et al. (2016). H-coil repetitive transcranial magnetic stimulation induced seizure in an adult with major depression: a case report. Brain Stimul. 9, 632-633. doi: 10.1016/j.brs.2016.04.013

Boggio, P. S., Zaghi, S., and Fregni, F. (2009). Modulation of emotions associated with images of human pain using anodal transcranial direct current stimulation (tDCS). Neuropsychologia 47, 212-217. doi: 10.1016/j.neuropsychologia.2008. 07.022

Bonavita, S., Sacco, R., Esposito, S., d'Ambrosio, A., Della Corte, M., Corbo, D., et al. (2017). Default mode network changes in multiple sclerosis: a link between depression and cognitive impairment? Eur. J. Neurol. 24, 27-36. doi: 10.1111/ene.13112

Brennan, S., McLoughlin, D. M., O’Connell, R., Bogue, J., O’Connor, S., McHugh, C., et al. (2017). Anodal transcranial direct current stimulation of the left dorsolateral prefrontal cortex enhances emotion recognition in 
depressed patients and controls. J. Clin. Exp. Neuropsychol. 39, 384-395. doi: 10.1080/13803395.2016.1230595

Bretlau, L. G., Lunde, M., Lindberg, L., Undén, M., Dissing, S., and Bech, P. (2008). Repetitive transcranial magnetic stimulation (rTMS) in combination with escitalopram in patients with treatment-resistant major depression: a double-blind, randomised, sham-controlled trial. Pharmacopsychiatry 41, 41-47. doi: 10.1055/s-2007-993210

Brunoni, A. R., Amadera, J., Berbel, B., Volz, M. S., Rizzerio, B. G., and Fregni, F. (2011). A systematic review on reporting and assessment of adverse effects associated with transcranial direct current stimulation. Int. J. Neuropsychopharmacol. 14, 1133-1145. doi: 10.1017/s1461145710001690

Brunoni, A. R., Valiengo, L., Baccaro, A., Zanão, T. A., de Oliveira, J. F., Goulart, A., et al. (2013). The sertraline vs. electrical current therapy for treating depression clinical study: results from a factorial, randomized, controlled trial. JAMA Psychiatry 70, 383-391. doi: 10.1001/2013.jamapsychiatry.32

Brunoni, A. R., Zanao, T. A., Vanderhasselt, M. A., Valiengo, L., de Oliveira, J. F., Boggio, P. S., et al. (2014). Enhancement of affective processing induced by bifrontal transcranial direct current stimulation in patients with major depression. Neuromodulation 17, 138-142. doi: 10.1111/ner.12080

Campbell, S., Marriott, M., Nahmias, C., and MacQueen, G. M. (2004). Lower hippocampal volume in patients suffering from depression: a meta-analysis. Am. J. Psychiatry 161, 598-607. doi: 10.1176/appi.ajp.161.4.598

Carle, G., Touat, M., Bruno, N., Galanaud, D., Peretti, C. S., Valero-Cabré, A., et al. (2017). Acute frontal lobe dysfunction following prefrontal low-frequency repetitive transcranial magnetic stimulation in a patient with treatmentresistant depression. Front. Psychiatry 8:96. doi: 10.3389/fpsyt.2017.00096

Carpenter, L. L., Aaronson, S. T., Clarke, G. N., Holtzheimer, P. E., Johnson, C. W., McDonald, W. M., et al. (2017). rTMS with a two-coil array: safety and efficacy for treatment resistant major depressive disorder. Brain Stimul. 10, 926-933. doi: 10.1016/j.brs.2017.06.003

Chen, A. C., and Etkin, A. (2013). Hippocampal network connectivity and activation differentiates post-traumatic stress disorder from generalized anxiety disorder. Neuropsychopharmacology $38,1889-1898$. doi: 10.1038/npp. 2013.122

Chen, J. J., Zhao, L. B., Liu, Y. Y., Fan, S. H., and Xie, P. (2017). Comparative efficacy and acceptability of electroconvulsive therapy versus repetitive transcranial magnetic stimulation for major depression: a systematic review and multiple-treatments meta-analysis. Behav. Brain Res. 320, 30-36. doi: 10.1016/j.bbr.2016.11.028

Clark, L., Chamberlain, S. R., and Sahakian, B. J. (2009). Neurocognitive mechanisms in depression: implications for treatment. Annu. Rev. Neurosci. 32, 57-74. doi: 10.1146/annurev.neuro.31.060407.125618

Collins, P. Y., Patel, V., Joestl, S. S., March, D., Insel, T. R., Daar, A. S., et al. (2011). Grand challenges in global mental health. Nature 475, 27-30. doi: $10.1038 / 475027 \mathrm{a}$

Conson, M., Errico, D., Mazzarella, E., Giordano, M., Grossi, D., and Trojano, L. (2015). Transcranial electrical stimulation over dorsolateral prefrontal cortex modulates processing of social cognitive and affective information. PLoS One 10:e0126448. doi: 10.1371/journal.pone. 0126448

Dalgleish, T. (2004). The emotional brain. Nat. Rev. Neurosci. 5, 583-589. doi: $10.1038 / \mathrm{nrn} 1432$

Davey, C. G., Allen, N. B., Harrison, B. J., and Yücel, M. (2011). Increased amygdala response to positive social feedback in young people with major depressive disorder. Biol. Psychiatry 69, 734-741. doi: 10.1016/j.biopsych.2010.12.004

Del Piero, L. B., Saxbe, D. E., and Margolin, G. (2016). Basic emotion processing and the adolescent brain: task demands, analytic approaches, and trajectories of changes. Dev. Cogn. Neurosci. 19, 174-189. doi: 10.1016/j.dcn.2016. 03.005

De Raedt, R., Vanderhasselt, M. A., and Baeken, C. (2015). Neurostimulation as an intervention for treatment resistant depression: from research on mechanisms towards targeted neurocognitive strategies. Clin. Psychol. Rev. 41, 61-69. doi: 10.1016/j.cpr.2014.10.006

Disner, S. G., Beevers, C. G., Haigh, E. A., and Beck, A. T. (2011). Neural mechanisms of the cognitive model of depression. Nat. Rev. Neurosci. 12, 467-477. doi: 10.1038/nrn3027

Dobek, C. E., Blumberger, D. M., Downar, J., Daskalakis, Z. J., and VilaRodriguez, F. (2015). Risk of seizures in transcranial magnetic stimulation: a clinical review to inform consent process focused on bupropion. Neuropsychiatr. Dis. Treat. 11, 2975-2987. doi: 10.2147/ndt.s91126

Downar, J., and Daskalakis, Z. J. (2013). New targets for rTMS in depression: a review of convergent evidence. Brain Stimul. 6, 231-240. doi: 10.1016/j.brs.2012.08.006

Downar, J., Geraci, J., Salomons, T. V., Dunlop, K., Wheeler, S., McAndrews, M. P., et al. (2014). Anhedonia and reward-circuit connectivity distinguish nonresponders from responders to dorsomedial prefrontal repetitive transcranial magnetic stimulation in major depression. Biol. Psychiatry 76, 176-185. doi: 10.1016/j.biopsych.2013.10.026

Erickson, K., Drevets, W. C., Clark, L., Cannon, D. M., Bain, E. E., Zarate, C. A., et al. (2005). Mood-congruent bias in affective go/no-go performance of unmedicated patients with major depressive disorder. Am. J. Psychiatry 162, 2171-2173. doi: 10.1176/appi.ajp.162.11.2171

Fernie, G., Bennett, D. M., Currie, J., Perrin, J. S., and Reid, I. C. (2014). Detecting objective and subjective cognitive effects of electroconvulsive therapy: intensity, duration and test utility in a large clinical sample. Psychol. Med. 44, 2985-2994. doi: $10.1017 / \mathrm{s} 0033291714000658$

Fox, M. D., Buckner, R. L., White, M. P., Greicius, M. D., and Pascual-Leone, A. (2012). Efficacy of transcranial magnetic stimulation targets for depression is related to intrinsic functional connectivity with the subgenual cingulate. Biol. Psychiatry 72, 595-603. doi: 10.1016/j.biopsych.2012.04.028

Galletly, C., Gill, S., Rigby, A., Carnell, B. L., and Clarke, P. (2016). Assessing the effects of repetitive transcranial magnetic stimulation on cognition in major depressive disorder using computerized cognitive testing. J. ECT 32, 169-173. doi: $10.1097 /$ yct. 0000000000000308

Garrett, A. S., and Maddock, R. J. (2006). Separating subjective emotion from the perception of emotion-inducing stimuli: an fMRI study. Neuroimage 33, 263-274. doi: 10.1016/j.neuroimage.2006.05.024

Gonda, X., Pompili, M., Serafini, G., Carvalho, A. F., Rihmer, Z., and Dome, P. (2015). The role of cognitive dysfunction in the symptoms and remission from depression. Ann. Gen. Psychiatry 14:27. doi: 10.1186/s12991-015-0068-9

Grimm, S., Beck, J., Schuepbach, D., Hell, D., Boesiger, P., Bermpohl, F., et al. (2008). Imbalance between left and right dorsolateral prefrontal cortex in major depression is linked to negative emotional judgment: an fMRI study in severe major depressive disorder. Biol. Psychiatry 63, 369-376. doi: 10.1016/j. biopsych.2007.05.033

Groenewold, N. A., Opmeer, E. M., de Jonge, P., Aleman, A., and Costafreda, S. G. (2013). Emotional valence modulates brain functional abnormalities in depression: evidence from a meta-analysis of fMRI studies. Neurosci. Biobehav. Rev. 37, 152-163. doi: 10.1016/j.neubiorev.2012.11.015

Hallett, M. (2007). Transcranial magnetic stimulation: a primer. Neuron 55, 187-199. doi: 10.1016/j.neuron.2007.06.026

Hamilton, J. P., Etkin, A., Furman, D. J., Lemus, M. G., Johnson, R. F., and Gotlib, I. H. (2012). Functional neuroimaging of major depressive disorder: a meta-analysis and new integration of base line activation and neural response data. Am. J. Psychiatry 169, 693-703. doi: 10.1176/appi.ajp.2012.110 71105

Hamilton, J. P., Siemer, M., and Gotlib, I. H. (2008). Amygdala volume in major depressive disorder: a meta-analysis of magnetic resonance imaging studies. Mol. Psychiatry 13, 993-1000. doi: 10.1038/mp.2008.57

Harmer, C. J., Goodwin, G. M., and Cowen, P. J. (2009). Why do antidepressants take so long to work? A cognitive neuropsychological model of antidepressant drug action. Br. J. Psychiatry 195, 102-108. doi: 10.1192/bjp.bp.108.051193

Herbsman, T., Avery, D., Ramsey, D., Holtzheimer, P., Wadjik, C., Hardaway, F., et al. (2009). More lateral and anterior prefrontal coil location is associated with better repetitive transcranial magnetic stimulation antidepressant response. Biol. Psychiatry 66, 509-515. doi: 10.1016/j.biopsych.2009.04.034

Holmes, A. J., and Pizzagalli, D. A. (2008). Response conflict and frontocingulate dysfunction in unmedicated participants with major depression. Neuropsychologia 46, 2904-2913. doi: 10.1016/j.neuropsychologia.2008. 05.028

Hoy, K. E., Segrave, R. A., Daskalakis, Z. J., and Fitzgerald, P. B. (2012) Investigating the relationship between cognitive change and antidepressant response following rTMS: a large scale retrospective study. Brain Stimul. 5, 539-546. doi: 10.1016/j.brs.2011.08.010

Huang, M. L., Luo, B. Y., Hu, J. B., Wang, S. S., Zhou, W. H., Wei, N., et al. (2012). Repetitive transcranial magnetic stimulation in combination with citalopram 
in young patients with first-episode major depressive disorder: a doubleblind, randomized, sham-controlled trial. Aust. N Z J. Psychiatry 46, 257-264. doi: $10.1177 / 0004867411433216$

Isenberg, K., Downs, D., Pierce, K., Svarakic, D., Garcia, K., Jarvis, M., et al. (2005). Low frequency rTMS stimulation of the right frontal cortex is as effective as high frequency rTMS stimulation of the left frontal cortex for antidepressantfree, treatment-resistant depressed patients. Ann. Clin. Psychiatry 17, 153-159. doi: 10.1080/10401230591002110

Jelovac, A., Kolshus, E., and McLoughlin, D. M. (2013). Relapse following successful electroconvulsive therapy for major depression: a metaanalysis. Neuropsychopharmacology 38, 2467-2474. doi: 10.1038/npp.20 13.149

Jha, S., Chadda, R. K., Kumar, N., and Bal, C. S. (2016). Brain SPECT guided repetitive transcranial magnetic stimulation (rTMS) in treatment resistant major depressive disorder. Asian J. Psychiatr. 21, 1-6. doi: 10.1016/j.ajp.2016. 02.003

Jin, X. L., Xu, W. Q., Le, Y. J., and Dai, X. K. (2016). Long-term effectiveness of modified electroconvulsive therapy compared with repetitive transcranial magnetic stimulation for the treatment of recurrent major depressive disorder. J. Nerv. Ment. Dis. 204, 479-482. doi: 10.1097/nmd.0000000000000493

Junghofer, M., Winker, C., Rehbein, M. A., and Sabatinelli, D. (2017). Noninvasive stimulation of the ventromedial prefrontal cortex enhances pleasant scene processing. Cereb. Cortex 27, 3449-3456. doi: 10.1093/cercor/bhx073

Koenigs, M., and Grafman, J. (2009). The functional neuroanatomy of depression: distinct roles for ventromedial and dorsolateral prefrontal cortex. Behav. Brain Res. 201, 239-243. doi: 10.1016/j.bbr.2009.03.004

Krakowski, M. I., and Czobor, P. (2017). Proneness to aggression and its inhibition in schizophrenia: interconnections between personality traits, cognitive function and emotional processing. Schizophr. Res. 184, 82-87. doi: 10.1016/j.schres.2016.11.038

Kuo, M. F., Paulus, W., and Nitsche, M. A. (2014). Therapeutic effects of non-invasive brain stimulation with direct currents (tDCS) in neuropsychiatric diseases. Neuroimage 85, 948-960. doi: 10.1016/j.neuroimage.2013.05.117

Lefaucheur, J. P., Antal, A., Ayache, S. S., Benninger, D. H., Brunelin, J., Cogiamanian, F., et al. (2017). Evidence-based guidelines on the therapeutic use of transcranial direct current stimulation (tDCS). Clin. Neurophysiol. 128, 56-92. doi: 10.1016/j.clinph.2016.10.087

Lepping, P., Schönfeldt-Lecuona, C., Sambhi, R. S., Lanka, S. V., Lane, S., Whittington, R., et al. (2014). A systematic review of the clinical relevance of repetitive transcranial magnetic stimulation. Acta Psychiatr. Scand. 130, 326-341. doi: 10.1111/acps.12276

Leyman, L., De Raedt, R., Schacht, R., and Koster, E. H. (2007). Attentional biases for angry faces in unipolar depression. Psychol. Med. 37, 393-402. doi: 10.1017/s003329170600910x

Liu, C. C., Qian, X. Y., An, J. X., Yu, Z. L., Wu, J. P., Wen, H., et al. (2016). Electroconvulsive therapy under general anesthesia with cisatracurium, laryngeal mask airways, and bispectral index. J. ECT 32, 17-19. doi: 10.1097/yct. 0000000000000251

Luber, B. M., Davis, S., Bernhardt, E., Neacsiu, A., Kwapil, L., Lisanby, S. H., et al. (2017). Using neuroimaging to individualize TMS treatment for depression: toward a new paradigm for imaging-guided intervention. Neuroimage 148, 1-7. doi: 10.1016/j.neuroimage.2016.12.083

Machii, K., Cohen, D., Ramos-Estebanez, C., and Pascual-Leone, A. (2006). Safety of rTMS to non-motor cortical areas in healthy participants and patients. Clin. Neurophysiol. 117, 455-471. doi: 10.1016/j.clinph.2005.10.014

Magnezi, R., Aminov, E., Shmuel, D., Dreifuss, M., and Dannon, P. (2016). Comparison between neurostimulation techniques repetitive transcranial magnetic stimulation vs. electroconvulsive therapy for the treatment of resistant depression: patient preference and cost-effectiveness. Patient Prefer. Adherence 10, 1481-1487. doi: 10.2147/ppa.s105654

Maizey, L., Allen, C. P., Dervinis, M., Verbruggen, F., Varnava, A., Kozlov, M., et al. (2013). Comparative incidence rates of mild adverse effects to transcranial magnetic stimulation. Clin. Neurophysiol. 124, 536-544. doi: 10.1016/j.clinph. 2012.07.024

Matthews, S. C., Strigo, I. A., Simmons, A. N., Yang, T. T., and Paulus, M. P. (2008). Decreased functional coupling of the amygdala and supragenual cingulate is related to increased depression in unmedicated individuals with current major depressive disorder. J. Affect. Disord. 111, 13-20. doi: 10.1016/j.jad.2008.05.022
McLoughlin, D. M., Mogg, A., Eranti, S., Pluck, G., Purvis, R., Edwards, D., et al. (2007). The clinical effectiveness and cost of repetitive transcranial magnetic stimulation versus electroconvulsive therapy in severe depression: a multicentre pragmatic randomised controlled trial and economic analysis. Health Technol. Assess. 11, 1-54. doi: 10.3310/hta11240

McNeely, H. E., Lau, M. A., Christensen, B. K., and Alain, C. (2008). Neurophysiological evidence of cognitive inhibition anomalies in persons with major depressive disorder. Clin. Neurophysiol. 119, 1578-1589. doi: 10.1016/j. clinph.2008.03.031

Meron, D., Hedger, N., Garner, M., and Baldwin, D. S. (2015). Transcranial direct current stimulation (tDCS) in the treatment of depression: systematic review and meta-analysis of efficacy and tolerability. Neurosci. Biobehav. Rev. 57, 46-62. doi: 10.1016/j.neubiorev.2015.07.012

Milev, R. V., Giacobbe, P., Kennedy, S. H., Blumberger, D. M., Daskalakis, Z. J., Downar, J., et al. (2016). Canadian network for mood and anxiety treatments (CANMAT) 2016 Clinical guidelines for the management of adults with major depressive disorder: section 4. Neurostimulation treatments. Can. J. Psychiatry 61, 561-575. doi: 10.1177/0706743716660033

Miller, E. K., and Cohen, J. D. (2001). An integrative theory of prefrontal cortex function. Annu. Rev. Neurosci. 24, 167-202. doi: 10.1146/annurev.neuro.24.1. 167

Mir-Moghtadaei, A., Caballero, R., Fried, P., Fox, M. D., Lee, K., Giacobbe, P., et al. (2015). Concordance between beamF3 and MRI-neuronavigated target sites for repetitive transcranial magnetic stimulation of the left dorsolateral prefrontal cortex. Brain Stimul. 8, 965-973. doi: 10.1016/j.brs.2015.05.008

Mitchell, P. B., and Loo, C. K. (2006). Transcranial magnetic stimulation for depression. Aust. N Z J. Psychiatry 40, 406-413. doi: 10.1080/j.1440-1614.2006. 01816.x

Möbius, M., Lacomblé, L., Meyer, T., Schutter, D. J. L. G., Gielkens, T., Becker, E. S., et al. (2017). Repetitive transcranial magnetic stimulation modulates the impact of a negative mood induction. Soc. Cogn. Affect. Neurosci. 12, 526-533. doi: 10.1093/scan/nsw180

Mondino, M., Thiffault, F., and Fecteau, S. (2015). Does non-invasive brain stimulation applied over the dorsolateral prefrontal cortex non-specifically influence mood and emotional processing in healthy individuals? Front. Cell. Neurosci. 9:399. doi: 10.3389/fncel.2015.00399

Narumoto, J., Okada, T., Sadato, N., Fukui, K., and Yonekura, Y. (2001). Attention to emotion modulates fMRI activity in human right superior temporal sulcus. Cogn. Brain Res. 12, 225-231. doi: 10.1016/s0926-6410(01) 00053-2

Nitsche, M. A., Fricke, K., Henschke, U., Schlitterlau, A., Liebetanz, D., Lang, N., et al. (2003). Pharmacological modulation of cortical excitability shifts induced by transcranial direct current stimulation in humans. J. Physiol. 553, 293-301. doi: 10.1113/jphysiol.2003.049916

Nitsche, M. A., Koschack, J., Pohlers, H., Hullemann, S., Paulus, W., and Happe, S. (2012). Effects of frontal transcranial direct current stimulation on emotional state and processing in healthy humans. Front. Psychiatry 3:58. doi: $10.3389 /$ fpsyt.2012.00058

Nitsche, M. A., and Paulus, W. (2000). Excitability changes induced in the human motor cortex by weak transcranial direct current stimulation. J. Physiol. 527, 633-639. doi: 10.1111/j.1469-7793.2000.t01-1-00633.x

Noda, Y., Silverstein, W. K., Barr, M. S., Vila-Rodriguez, F., Downar, J., Rajji, T. K., et al. (2015). Neurobiological mechanisms of repetitive transcranial magnetic stimulation of the dorsolateral prefrontal cortex in depression: a systematic review. Psychol. Med. 45, 3411-3432. doi: 10.1017/s00332917150 01609

Otte, C., Gold, S. M., Penninx, B. W., Pariante, C. M., Etkin, A., Fava, M., et al. (2016). Major depressive disorder. Nat. Rev. Dis. Primers 2:16065. doi: $10.1038 /$ nrdp. 2016.65

Pessoa, L. (2017). A network model of the emotional brain. Trends Cogn. Sci. 21, 357-371. doi: 10.1016/j.tics.2017.03.002

Prete, G., Laeng, B., Fabri, M., Foschi, N., and Tommasi, L. (2015). Right hemisphere or valence hypothesis, or both? The processing of hybrid faces in the intact and callosotomized brain. Neuropsychologia 68, 94-106. doi: 10.1016/j.neuropsychologia.2015.01.002

Prikryl, R., and Kucerova, H. (2005). Occurrence of epileptic paroxysm during repetitive transcranial magnetic stimulation treatment. J. Psychopharmacol. 19:313. doi: $10.1177 / 0269881105051545$ 
Raichle, M. E., MacLeod, A. M., Snyder, A. Z., Powers, W. J., Gusnard, D. A., and Shulman, G. L. (2001). A default mode of brain function. Proc. Natl. Acad. Sci. U S A 98, 676-682. doi: 10.1073/pnas.98.2.676

Roiser, J. P., Elliott, R., and Sahakian, B. J. (2012). Cognitive mechanisms of treatment in depression. Neuropsychopharmacology 37, 117-136. doi: $10.1038 /$ npp. 2011.183

Rossini, D., Magri, L., Lucca, A., Giordani, S., Smeraldi, E., and Zanardi, R. (2005). Does rTMS hasten the response to escitalopram, sertraline, or venlafaxine in patients with major depressive disorder? A double-blind, randomized, sham-controlled trial. J. Clin. Psychiatry 66, 1569-1575. doi: 10.4088/jcp. v66n 1212

Rumi, D. O., Gattaz, W. F., Rigonatti, S. P., Rosa, M. A., Fregni, F., Rosa, M. O., et al. (2005). Transcranial magnetic stimulation accelerates the antidepressant effect of amitriptyline in severe depression: a double-blind placebo-controlled study. Biol. Psychiatry 57, 162-166. doi: 10.1016/j.biopsych.2004.10.029

Rush, A. J., Kraemer, H. C., Sackeim, H. A., Fava, M., Trivedi, M. H., Frank, E., et al. (2006). Report by the ACNP Task Force on response and remission in major depressive disorder. Neuropsychopharmacology 31, 1841-1853. doi: 10.1038/sj.npp.1301131

Sanchez, A., Vanderhasselt, M. A., Baeken, C., and De Raedt, R. (2016). Effects of tDCS over the right DLPFC on attentional disengagement from positive and negative faces: an eye-tracking study. Cogn. Affect. Behav. Neurosci. 16, 1027-1038. doi: 10.3758/s13415-016-0450-3

Schutter, D. J., and van Honk, J. (2005). A framework for targeting alternative brain regions with repetitive transcranial magnetic stimulation in the treatment of depression. J. Psychiatry Neurosci. 30, 91-97.

Serafini, G., Pompili, M., Belvederi Murri, M., Respino, M., Ghio, L., Girardi, P., et al. (2015). The effects of repetitive transcranial magnetic stimulation on cognitive performance in treatment-resistant depression. A systematic review. Neuropsychobiology 71, 125-139. doi: 10.1159/000381351

Shiozawa, P., da Silva, M. E., and Cordeiro, Q. (2015). Transcranial direct current stimulation for treating depression in a patient with right hemispheric dominance: a case study. J. ECT 31, 201-202. doi: 10.1097/yct. 0000000000000180

Shiozawa, P., Fregni, F., Bensenor, I. M., Lotufo, P. A., Berlim, M. T., Daskalakis, J. Z., et al. (2014). Transcranial direct current stimulation for major depression: an updated systematic review and meta-analysis. Int. J. Neuropsychopharmacol. 17, 1443-1452. doi: 10.1017/s1461145714000418

Stagg, C. J., and Nitsche, M. A. (2011). Physiological basis of transcranial direct current stimulation. Neuroscientist 17, 37-53. doi: 10.1177/1073858410386614

Strunk, D. R., and Adler, A. D. (2009). Cognitive biases in three prediction tasks: a test of the cognitive model of depression. Behav. Res. Ther. 47, 34-40. doi: 10.1016/j.brat.2008.10.008

Teng, S., Guo, Z., Peng, H., Xing, G., Chen, H., He, B., et al. (2017). Highfrequency repetitive transcranial magnetic stimulation over the left DLPFC for major depression: session-dependent efficacy: a meta-analysis. Eur. Psychiatry 41, 75-84. doi: 10.1016/j.eurpsy.2016.11.002

Tortella, G., Selingardi, P. M., Moreno, M. L., Veronezi, B. P., and Brunoni, A. R. (2014). Does non-invasive brain stimulation improve cognition in major depressive disorder? A systematic review. CNS Neurol. Disord. Drug Targets 13, 1759-1769. doi: 10.2174/18715273136661411302 24431

UK ECT Review Group. (2003). Efficacy and safety of electroconvulsive therapy in depressive disorders: a systematic review and meta-analysis. Lancet 361, 799-808. doi: 10.1016/s0140-6736(03)12705-5

Utz, K. S., Dimova, V., Oppenländer, K., and Kerkhoff, G. (2010). Electrified minds: transcranial direct current stimulation (tDCS) and galvanic vestibular stimulation (GVS) as methods of non-invasive brain stimulation in neuropsychology - a review of current data and future implications. Neuropsychologia 48, 2789-2810. doi: 10.1016/j.neuropsychologia.2010. 06.002
Valero-Cabré, A., Amengual, J., Stengel, C., Pascual-Leone, A., and Coubard, O. A. (2017). Transcranial magnetic stimulation in basic and clinical neuroscience: a comprehensive review of fundamental principles and novel insights. Neurosci. Biobehav. Rev. doi: 10.1016/j.neubiorev.2017.10.006 [Epub ahead of print].

Vanderhasselt, M. A., De Raedt, R., Baeken, C., Leyman, L., and D’Haenen, H. (2009). A single session of rTMS over the left dorsolateral prefrontal cortex influences attentional control in depressed patients. World J. Biol. Psychiatry 10, 34-42. doi: 10.1080/15622970701816514

Vigod, S., Dennis, C. L., Daskalakis, Z., Murphy, K., Ray, J., Oberlander, T., et al. (2014). Transcranial direct current stimulation (tDCS) for treatment of major depression during pregnancy: study protocol for a pilot randomized controlled trial. Trials 15:366. doi: 10.1186/1745-6215-15-366

Wall, C., Croarkin, P., Bandel, L., and Schaefer, K. (2014). Response to repetitive transcranial magnetic stimulation induced seizures in an adolescent patient with major depression: a case report. Brain Stimul. 7, 337-338. doi: 10.1016/j. brs.2013.12.001

Wang, Y. Y., Jiang, N. Z., Cheung, E. F., Sun, H. W., and Chan, R. C. (2015). Role of depression severity and impulsivity in the relationship between hopelessness and suicidal ideation in patients with major depressive disorder. J. Affect. Disord. 183, 83-89. doi: 10.1016/j.jad.2015. 05.001

Wang, Y. M., Li, N., Yang, L. L., Song, M., Shi, L., Chen, W. H., et al. (2017). Randomized controlled trial of repetitive transcranial magnetic stimulation combined with paroxetine for the treatment of patients with first-episode major depressive disorder. Psychiatry Res. 254, 18-23. doi: 10.1016/j.psychres.2017. 04.005

Whiteford, H. A., Degenhardt, L., Rehm, J., Baxter, A. J., Ferrari, A. J., Erskine, H. E., et al. (2013). Global burden of disease attributable to mental and substance use disorders: findings from the Global Burden of Disease Study 2010. Lancet 382, 1575-1586. doi: 10.1016/S0140-6736(13) 61611-6

Wolkenstein, L., and Plewnia, C. (2013). Amelioration of cognitive control in depression by transcranial direct current stimulation. Biol. Psychiatry 73, 646-651. doi: 10.1016/j.biopsych.2012.10.010

Wolkenstein, L., Kanske, P., Bailer, J., Wessa, M., Hautzinger, M., and Joormann, J. (2017). Impaired cognitive control over emotional material in euthymic bipolar disorder. J. Affect. Disord. 214, 108-114. doi: 10.1016/j.jad.2017. 03.007

Yang, W., Zhu, X., Wang, X., Wu, D., and Yao, S. (2011). Time course of affective processing bias in major depression: an ERP study. Neurosci. Lett. 487, 372-377. doi: 10.1016/j.neulet.2010.10.059

Zilverstand, A., Parvaz, M. A., and Goldstein, R. Z. (2017). Neuroimaging cognitive reappraisal in clinical populations to define neural targets for enhancing emotion regulation. A systematic review. Neuroimage 151, 105-116. doi: 10.1016/j.neuroimage.2016.06.009

Zwanzger, P., Steinberg, C., Rehbein, M. A., Bröckelmann, A. K., Dobel, C., Zavorotnyy, M., et al. (2014). Inhibitory repetitive transcranial magnetic stimulation (rTMS) of the dorsolateral prefrontal cortex modulates early affective processing. Neuroimage 101, 193-203. doi: 10.1016/j.neuroimage. 2014.07.003

Conflict of Interest Statement: The authors declare that the research was conducted in the absence of any commercial or financial relationships that could be construed as a potential conflict of interest.

Copyright (C) 2017 Liu, Sheng, Li and Zhang. This is an open-access article distributed under the terms of the Creative Commons Attribution License (CC BY). The use, distribution or reproduction in other forums is permitted, provided the original author(s) or licensor are credited and that the original publication in this journal is cited, in accordance with accepted academic practice. No use, distribution or reproduction is permitted which does not comply with these terms. 